OPEN ACCESS

Edited by:

Tomer Avidor-Reiss,

The University of Toledo,

United States

Reviewed by:

Xi-Feng Zhang,

Wuhan Polytechnic University, China

Myung-Geol Pang,

Chung-Ang University, South Korea

${ }^{*}$ Correspondence:

Polina V. Lishko

lishko@berkeley.edu

Specialty section:

This article was submitted to

Cell Growth and Division,

a section of the journal

Frontiers in Cell and Developmental

Biology

Received: 10 March 2020

Accepted: 07 May 2020

Published: 30 June 2020

Citation:

Khasin LG, Della Rosa J,

Petersen N, Moeller J, Kriegsfeld LJ and Lishko PV (2020) The Impact of Di-2-Ethylhexyl Phthalate on Sperm Fertility. Front. Cell Dev. Biol. 8:426.

doi: 10.3389/fcell.2020.00426

\section{The Impact of Di-2-Ethylhexyl Phthalate on Sperm Fertility}

\author{
Liliya Gabelev Khasin', John Della Rosa1,2, Natalie Petersen', Jacob Moeller ${ }^{3}$, \\ Lance J. Kriegsfeld ${ }^{3,4,5,6}$ and Polina V. Lishko ${ }^{1,3 *}$
}

\begin{abstract}
'Department of Molecular \& Cell Biology, University of California, Berkeley, Berkeley, CA, United States, ${ }^{2}$ Department of Chemistry, University of California, Berkeley, Berkeley, CA, United States, ${ }^{3}$ Graduate Group in Endocrinology, University of California, Berkeley, Berkeley, CA, United States, ${ }^{4}$ Department of Integrative Biology, University of California, Berkeley, Berkeley, CA, United States, ${ }^{5}$ Department of Psychology, University of California, Berkeley, Berkeley, CA, United States,

${ }^{6}$ Helen Wills Neuroscience Institute, University of California, Berkeley, Berkeley, CA, United States
\end{abstract}

A growing number of studies point to reduced fertility upon chronic exposure to endocrine-disrupting chemicals (EDCs) such as phthalates and plasticizers. These toxins are ubiquitous and are often found in food and beverage containers, medical devices, as well as in common household and personal care items. Animal studies with EDCs, such as phthalates and bisphenol A have shown a dose-dependent decrease in fertility and embryo toxicity upon chronic exposure. However, limited research has been conducted on the acute effects of these EDCs on male fertility. Here we used a murine model to test the acute effects of four ubiquitous environmental toxins: bisphenol $A$ (BPA), di-2-ethylhexyl phthalate (DEHP), diethyl phthalate (DEP), and dimethyl phthalate (DMP) on sperm fertilizing ability and pre-implantation embryo development. The most potent of these toxins, di-2-ethylhexyl phthalate (DEHP), was further evaluated for its effect on sperm ion channel activity, capacitation status, acrosome reaction and generation of reactive oxygen species (ROS). DEHP demonstrated a profound hazardous effect on sperm fertility by producing an altered capacitation profile, impairing the acrosome reaction, and, interestingly, also increasing ROS production. These results indicate that in addition to its known chronic impact on reproductive potential, DEHP also imposes acute and profound damage to spermatozoa, and thus, represents a significant risk to male fertility.

Keywords: endocrine-disrupting chemicals (EDC), di-2-ethylhexyl phthalate (DEHP), phthalates, spermatozoa, capacitation, embryo development, acrosome reaction, reactive oxygen species (ROS)

\section{INTRODUCTION}

Phthalates and plasticizers are synthetic chemicals that are utilized to make plastic more flexible. They are known to act as endocrine-disrupting chemicals (EDC) (Rudel et al., 2003; Hunt et al., 2009), which are ubiquitous in food and beverage containers, as well as coatings of pills, medical tubing (Green et al., 2005; Hauser and Calafat, 2005) and plastic packaging (Muncke, 2011). Phthalates and plasticizers are bound to plastic polymers by non-covalent bonds, and thus, easily leak into the environment (Pearson and Trissel, 1993). The main routes of exposure to these substances are ingestion, inhalation, dermal absorption, or intravenous medication administration (Hauser and Calafat, 2005; Meeker et al., 2009). Consequently, the vast majority of the population 
is exposed to these toxins on a daily basis. Low micromolar concentrations of certain EDCs in human urine, sweat and plasma have been associated with an increased rate of miscarriages and compromised male and female fertility (Lovekamp and Davis, 2001; Duty et al., 2003; Hauser et al., 2006; Svechnikova et al., 2007; Burdorf et al., 2011; Toft et al., 2012; Wang et al., 2012; Bloom et al., 2015; Patel et al., 2015; Brehm and Flaws, 2019).

In the present study, we evaluated the reproductive outcomes of acute exposure to four omnipresent EDCs - Bisphenol A (BPA), dimethyl phthalate (DMP), diethyl phthalate (DEP) and Di-ethyl hexyl phthalate (DEHP). BPA, a plasticizer manufactured in large volumes for the production of polycarbonate plastics and epoxy resins, is used to line food and beverage storage containers, coat water supply pipes and is also a component of dental fillings. The aforementioned exposure routes lead to detectable levels of BPA in human serum (Vandenberg et al., 2007), urine (Calafat et al., 2005; Hauser et al., 2007), adipose tissue (Fernandez et al., 2007) and breast milk (Sun et al., 2004). Numerous studies have shown that chronic exposure to BPA alters fertility in both males and females (Zota et al., 2014; Zhou et al., 2017).

Phthalates are similarly produced in large volumes and are used as plasticizing agents. Phthalates can be grouped into two broad categories: low-molecular-weight and high-molecularweight phthalates. The low-molecular-weight phthalates, such as DEP and DMP, are commonly found in cosmetics and personal care products, respectively. Specifically, DEP is utilized as a solvent and a fixative in fragrances. Studies have shown that chronic exposure to DEP may lead to multigenerational effects on reproductive health in both male and female rats (Fujii et al., 2005). The second most common low-molecular-weight phthalate is DMP. DMP is used primarily as an insect repellent, resulting in extensive exposure due to generous application to exposed skin and clothing. A recent study in mice found that chronic exposure to DMP triggers changes in the levels of serum hormone that lead to increased rates of ovarian granulosa cell death (Mei et al., 2019).

The high-molecular-weight phthalates, such as Di-ethyl hexyl phthalate (DEHP), are used in construction materials and numerous polyvinyl chloride (PVC) products. DEHP is one of the most commonly used phthalates (Wang et al., 2019) and is of primary interest regarding its disrupting impact on fertility. In fact, $98 \%$ of the US population test positive for DEHP and its metabolites (Api, 2001; Zota et al., 2016). Despite numerous reports on its toxicity, DEHP is still widely used in consumer products and in a number of medical devices, such as blood bags, infusion tubes, nasogastric tubes, peritoneal dialysis bags, and urological catheters. Patients who undergo frequent hemodialysis, catheterization or massive blood transfusions are at particular risk for DEHP toxicity and are exposed to doses as high as $168 \mathrm{mg} /$ day (Kavlock et al., 2002). Several human studies have demonstrated the profound effects of prolonged exposure to DEHP on both male and female fertility (Burdorf et al., 2011; Pan et al., 2011; Sumner et al., 2019).

The majority of studies on EDC's impact on reproductive health, including those mentioned above, evaluated the toxic effects of chronic exposure to phthalates and plasticizers. However, little is known about the reproductive outcomes of short exposure to such EDCs.

In the present study, we assessed the effects of acute exposure to BPA, DMP, DEP, and DEHP. Out of the four EDCs tested, DEHP demonstrated the strongest effect on male fertility by significantly altering the maturation process sperm undergoes, also known as capacitation, as well as inhibiting acrosome reaction, and triggering excessive reactive oxygen species (ROS) production. Altogether these changes led to sperm inability to fertilize eggs. These results suggest that DEHP can directly affect sperm fertility and is therefore detrimental to male reproductive health.

\section{RESULTS}

\section{Murine Embryo Development Is Impacted by DMP, BPA, DEP, and DEHP}

Exposure to phthalates could either damage sperm directly or impair pre-implantation embryo development after fertilization occurs. To test the susceptibility of pre-implanted embryos to DEHP, DMP, DEP, and BPA, naturally derived zygotes were harvested and subjected to $0,1,2$, and $10 \mu \mathrm{M}$ of each EDC as outlined in the methods section. The ability of the pre-implantation embryo to progress toward the blastocyst stage was recorded on day 5 post-fertilization (Figure 1 and Supplementary Figure S1), and the respective survival rate was calculated as described in methods. While all four tested compounds did not affect pre-implantation development at the lower concentrations (up to $2 \mu \mathrm{M}$ ), we found that at $10 \mu \mathrm{M}$, all four chemicals effectively prevented blastocyst formation ( $p<0.05$; Figures 1A-H and Supplementary Tables S1A-D). All controls have been performed with either vehicle control $(0.1 \%$ ethanol) or EDC-free media. No significant differences were observed among control conditions (Supplementary Table S2).

\section{In vitro Fertilization Is Affected by DEHP}

To explore the direct effect of DEHP, DMP, DEP, and BPA on sperm fertilizing ability, in vitro fertilization (IVF) assays were carried out. Mouse sperm were capacitated in phthalatesupplemented media as described in methods by exposing sperm to different concentrations $(0,1,2$, and $10 \mu \mathrm{M})$ of EDCs, and 60-90 min post-exposure, sperm was introduced to healthy murine eggs. The fertilization rate was calculated and presented as the percentage of embryos that reached the morula or blastula stage on day 5 post-fertilization $(p<0.05$; Figures $\mathbf{2 A}-\mathbf{H}$, Supplementary Figure S2, and Supplementary Tables S3AD). As shown in Figure 2, all tested concentrations of DEP and DMP did not produce any effect on sperm fertilizing ability and subsequent blastocyst formation, while $10 \mu \mathrm{M}$ BPA had a minimal, but not a statistically significant impact on early embryo development (Figures 2C,D and Supplementary Figure S2). The most damaging effect to blastocyst formation was observed with DEHP (Figures 2G,H). While spermatozoa retained their fertilization potential at $1 \mu \mathrm{M}$, a significant decrease in embryo progression to blastulae was found already at 
A

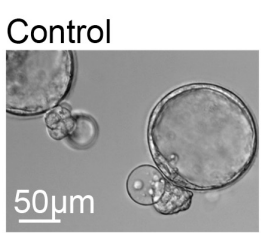

C

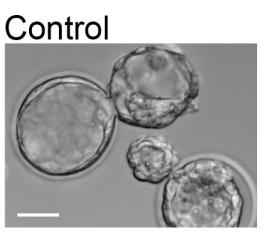

E Control

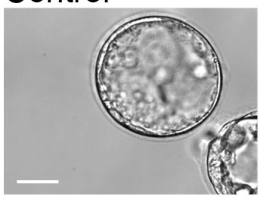

G

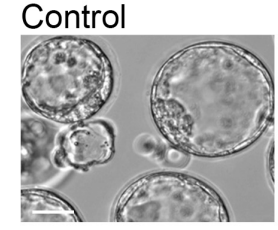

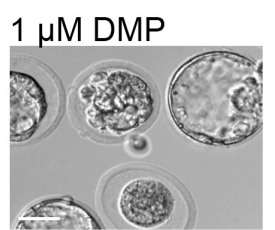
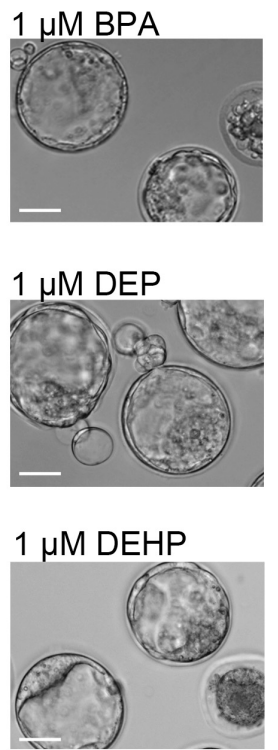
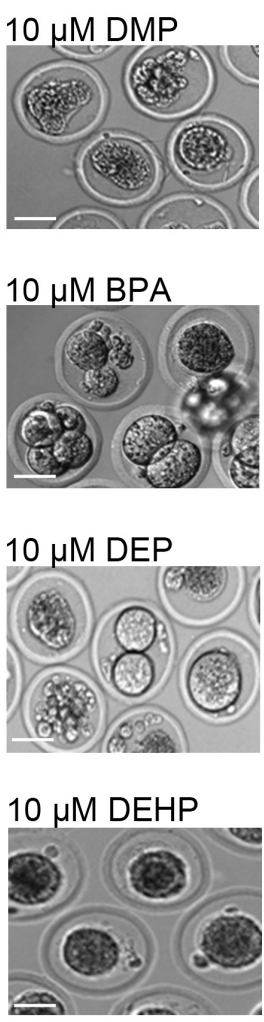
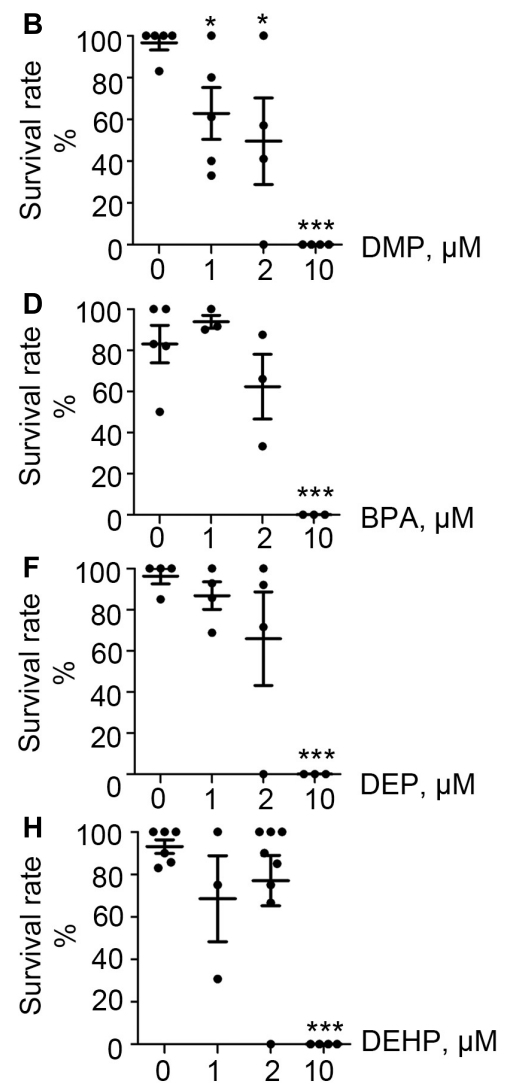

FIGURE 1 | Murine embryo development is impacted by DMP, BPA, DEP and DEHP. In vitro embryo development on day 5 post fertilization. Panels (A,C,E,G) are representative images of blastocysts previously exposed at the zygote stage to 0, 1, or $10 \mu \mathrm{M}$ of the indicated EDC for $20 \mathrm{~h}$. The subsequent embryo culture was done in the absences of the indicated EDC. (A) Shown are representative images of DMP-exposed embryos. (B) The survival rate of DMP exposed zygotes was calculated based on the percentage of embryos that have reached the morula or blastocyst stage. (C) Representative images of embryos previously exposed to BPA. (D) The survival rate of BPA exposed zygotes was calculated as in (B). (E) Representative images of embryos previously exposed to DEP. (F) The survival rate of DEP exposed zygotes was calculated as in (B). (G) Representative images of embryos previously exposed to DEHP. (H) The survival rate of DEHP- exposed zygotes was calculated as in (B). Data are means \pm S.E.M. Asterisk indicates a statistical difference between control embryos and embryos exposed to EDCs. ${ }^{\star} P \leq 0.05,{ }^{\star \star} P<0.01,{ }^{\star \star \star} P<0.001$. Scale bars for all images are $50 \mu \mathrm{m}$.

$2 \mu \mathrm{M}(74.95 \pm 5.459 \%$ in control vs. $47.68 \pm 9.68 \%$ in $2 \mu \mathrm{M})$. Moreover, at $10 \mu \mathrm{M}$ of DEHP, almost no blastocyst formation was observed (Figures 2G,H). All controls have been done with either $0.1 \%$ ethanol as a vehicle control or EDC-free media, and no significant differences were detected between the control conditions (Supplementary Table S4).

\section{DEHP Prevents Fertilization}

The acute exposure to DEHP may either affect sperm ability to fertilize the egg or may permit fertilization but subsequently inhibit the zygotic division. To distinguish between these two scenarios, sperm fertility was assessed by recording pronuclei formation $9 \mathrm{~h}$ post IVF. As shown in Figure 3, in the presence of $10 \mu \mathrm{M}$ DEHP, a $92.76 \%$ reduction in pronuclei formation was detected compared to untreated control. Values were calculated based on pooled data from three independent experiments and represent a total of 19 zygotes out of 25 eggs in the control conditions, versus 2 zygotes out of 36 eggs in the presence of DEHP (Supplementary Table S5). These results indicate that even short exposure to DEHP modifies sperm physiology making spermatozoa unable to penetrate the zona pellucida.

\section{Murine CatSper Is Not Affected by DEHP}

Since DEHP has demonstrated the most substantial effect on sperm fertility among all tested EDCs, we further explored which sperm functions were directly affected by exposure to this phthalate. Once deposited inside the female reproductive tract, mammalian spermatozoa must undergo a final maturation step, i.e., capacitation, to become competent to fertilize the egg (Austin, 1951; Yanagimachi, 1994). This process results in the removal of non-covalently attached glycoproteins, depletion of cholesterol, and other steroids (Davis, 1981), as well as the removal of adherent seminal plasma proteins (Chang, 1957). These physiological changes alter sperm membrane potential and make the cell competent to undergo a change in motility, known as hyperactivation, trigger the acrosome reaction and prepare spermatozoa for fertilization. Hyperactivation is characterized by calcium influx into the sperm flagellum via the calcium 


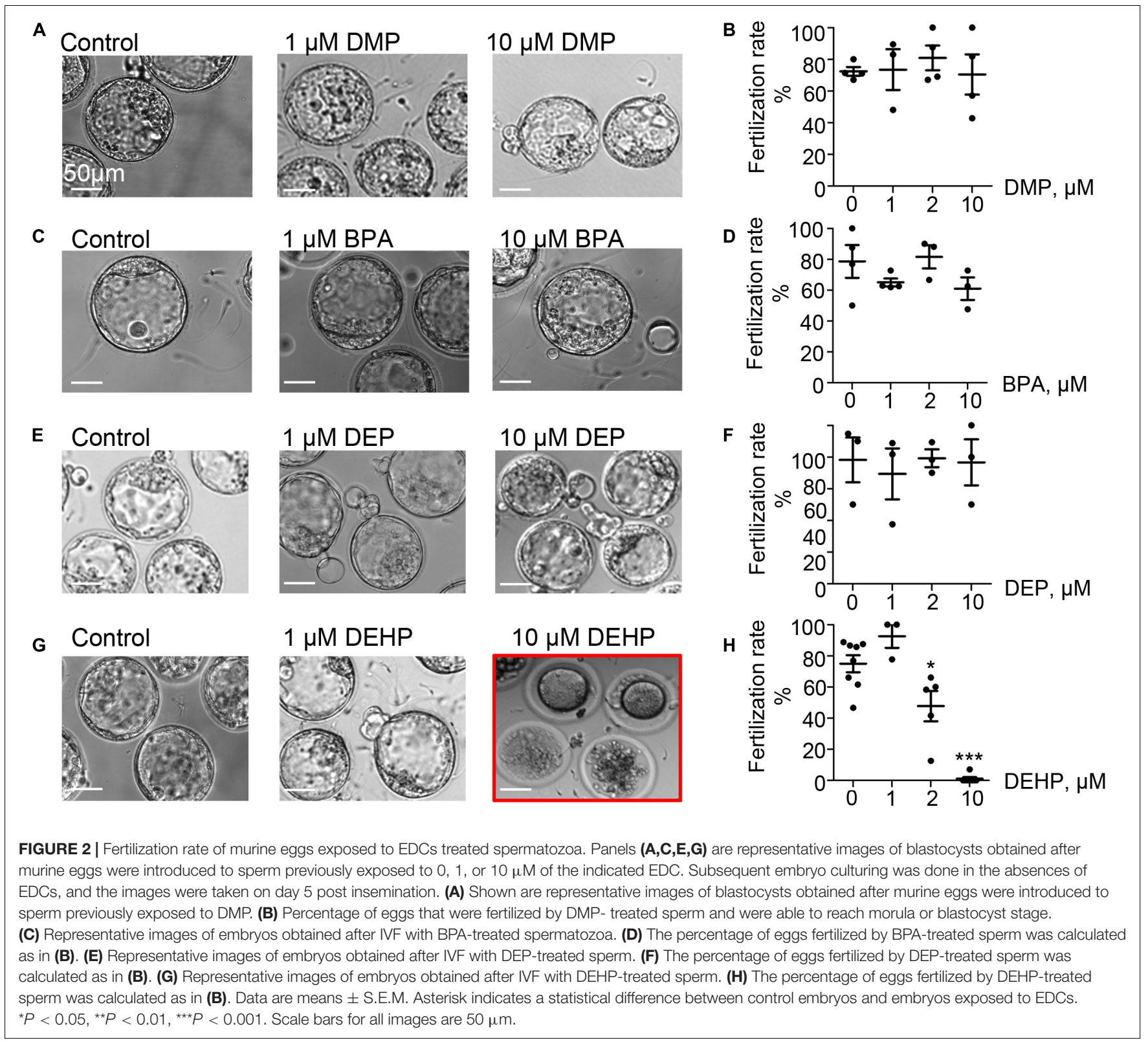

channel- CatSper (Ren et al., 2001; Carlson et al., 2003) and is defined as an asymmetrical flagellar beat that is required for penetration through the viscous luminal fluids of the female reproductive tract and the protective vestments of the egg. CatSper deficiency, as well as its suppression by environmental toxins, has been previously linked to male infertility (Ren et al., 2001; Qi et al., 2007; Schiffer et al., 2014; Tamburrino et al., 2014). To investigate whether murine CatSper is also affected by DEHP, we used murine sperm patch-clamp technique (Kirichok et al., 2006). As shown in Supplementary Figures S3A,B, the application of $10 \mu \mathrm{M}$ DEHP did not alter CatSper currents. Since previous studies on several EDCs reported that phthalates impact human CatSper at micromolar concentrations (Schiffer et al., 2014), we also tested DEHP at a higher dose (Supplementary Figures S3A,B). However, at either 10 or $100 \mu \mathrm{M}$ concentration, no significant changes in monovalent CatSper currents were observed. This indicates that DEHP affects sperm cells through a CatSper-independent mechanism.

\section{DEHP Alters Sperm Capacitation and ROS Production}

Another hallmark of capacitation is the phosphorylation of sperm proteins on tyrosine residues (Salicioni et al., 2007; Visconti, 2009). Previous reports on the capacitation of murine sperm demonstrate a time-dependent increase in the phosphorylation of tyrosine residues in proteins with the molecular weight of 40-170 kDa (Visconti et al., 1995a; Naz and Rajesh, 2004; Sepideh et al., 2009; Visconti, 2009). This modification allows sperm to hyperactivate, undergo the acrosome reaction 

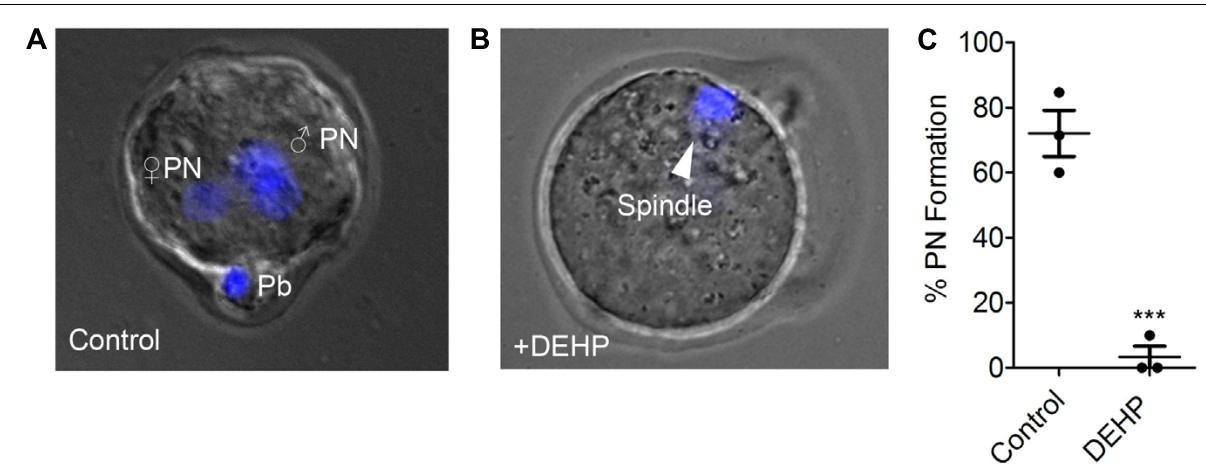

FIGURE 3 | Pronuclei formation after in vitro fertilization by DEHP-treated sperm. Pronuclear formation was assessed $9 \mathrm{~h}$ after IVF; genomic DNA staining was done with DAPI. (A) Representative image of a successfully fertilized egg with two pronuclei (PN) and a polar body (Pb). The egg was inseminated with sperm treated with vehicle control solution. (B) Shown are representative images of unfertilized eggs following an IVF with sperm that was treated with $10 \mu \mathrm{M}$ DEHP. The arrowhead indicates the position of the second metaphase spindle. (C) Percentage of fertilized eggs with PN detected. Each data point represents the mean of one of the three independent experiments \pm S.E.M. ${ }^{\star \star \star}$ indicates statistical significance $(P<0.005)$. The total number of eggs: 25 (control), 36 (+10 $\mu \mathrm{M}$ DEHP).

and interact with the zona pellucida (Nassar et al., 1999; Naz and Rajesh, 2004). To test the effect of DEHP on sperm tyrosine phosphorylation, caudal epididymal spermatozoa were incubated in a capacitating medium containing either $10 \mu \mathrm{M}$ DEHP or vehicle control. Subsequently, tyrosine phosphorylation was assessed by a western blot using a monoclonal antiphosphotyrosine antibody (anti-PY) (EMD Millipore). As shown in Figures 4A,B, DEHP markedly alters sperm capacitationassociated tyrosine phosphorylation kinetics, by expediting the process within the first $60 \mathrm{~min}$ of exposure, followed by a complete reversal after $120 \mathrm{~min}$ of incubation. Specifically, at the 60 -min time point, a $150 \%$ increase in the global tyrosine phosphorylation was detected in DEHP treated spermatozoa in comparison to the control condition. As capacitation progressed, the detected levels of the global tyrosine phosphorylation increased in the control samples. However, in the DEHP treated samples, the levels of detected phosphotyrosine significantly dropped. Specifically, at the 120-min time point, the detected levels of phosphoproteins were 59\% lower in the control. The most striking changes in phosphorylation were observed at the regions that correspond to $75,95,170$, and $270 \mathrm{kDa}$ (Supplementary Figure S4). The 75, 95, and $170 \mathrm{kDa}$ molecular weight proteins were previously reported to have important roles in sperm fertility (Naz and Rajesh, 2004; Sepideh et al., 2009). In addition, immunocytochemistry experiments using the same anti-PY antibody, revealed that the increased protein phosphorylation caused by DEHP is primarily localized to the mid-piece region of sperm (Figure 4C). According to previous reports, during the normal capacitation process, the sperm midpiece is undergoing more robust tyrosine phosphorylation in comparison to other parts of the flagellum (Alvau et al., 2016), and it appears that DEHP exacerbates this process. To further assess the global changes in sperm tyrosine phosphorylation induced by DEHP, flow cytometry analysis was performed using anti-PY labeled with CF647 dye (Biotium). As shown in Figure 4D, a significant increase in overall fluorescence was observed in DEHP treated cells resulting in 1.5- \pm 0.2 -fold increase in global fluorescence.
The mid-piece region of sperm flagellum harbors mitochondria- an organelle known to generate ROS. Interestingly, DEHP increases ROS generation in various cells and tissues, including hepatocytes, adipocytes, and testis (Kasahara et al., 2002; Schaedlich et al., 2018; Huang et al., 2019). However, DEHP's ability to alter sperm ROS production has not been studied. To detect ROS production, a chemiluminescence assay was employed, a commonly described technique to detect ROS in semen (Ochsendorf et al., 1994; Williams and Ford, 2005; Agarwal et al., 2008). The levels of ROS production were assessed in caudal sperm capacitated in the presence or absence of 10 and $100 \mu \mathrm{M}$ DEHP after 60 min of exposure. A significant increase in ROS production was detected in all treated samples in comparison to vehicle-treated controls (Figure 5A). We found that already at $10 \mu \mathrm{M}$ of DEHP, a maximal ROS production was achieved, and no further increase was detected at $100 \mu \mathrm{M}$. Since excessive ROS production is known to be cytotoxic to sperm, exposure to DEHP may lead to impaired sperm fertilizing capacity.

\section{The Acrosome Reaction in Capacitated Spermatozoa Is Inhibited by DEHP}

The acrosome reaction is the fusion of the sperm plasma membrane with the outer acrosomal membrane. It is vital for fertilization, as only acrosome-reacted spermatozoa can fuse with the egg (Avella and Dean, 2011). According to previous reports, increased levels of ROS production result in excessive peroxidation of the sperm acrosomal membrane (Zalata et al., 2004), impairing acrosomal exocytosis and sperm-egg fusion (Aitken and Clarkson, 1987; Griveau et al., 1995; Ichikawa et al., 1999). While there is a debate over the physiological triggers for the acrosome reaction and the exact site of acrosomal exocytosis, it is well accepted that the acrosome reaction is required for sperm fertility. Therefore, we have explored whether DEHP can alter the spontaneous acrosome reaction. We found that incubation with $10 \mu \mathrm{M}$ DEHP, decreased the percentage of spontaneous acrosome reaction. In control samples, the detected rates of acrosome 


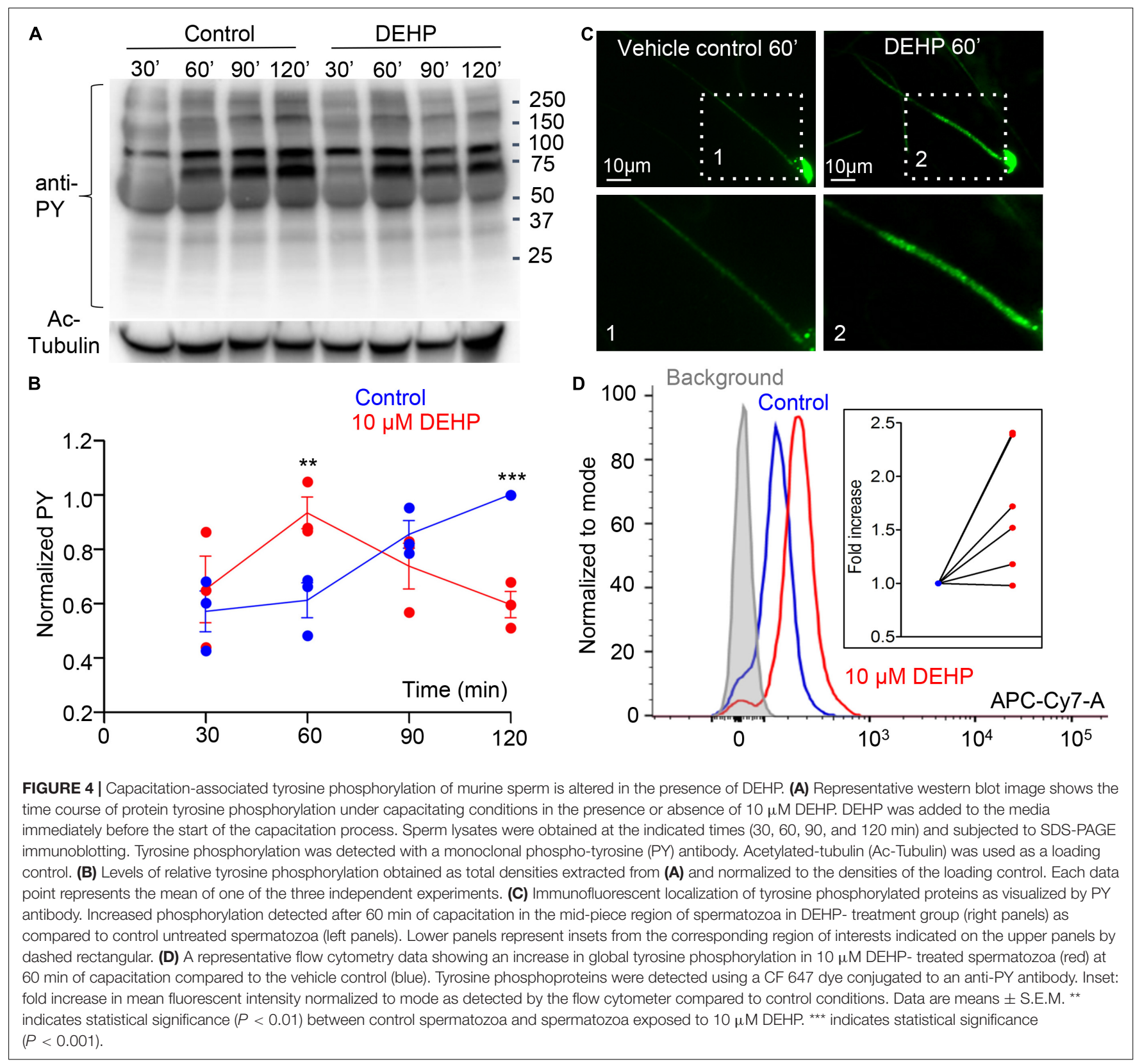

reacted cells were $20.95 \pm 0.62 \%$, whereas in DEHP treated samples, the rates dropped to $8.28 \pm 1.24 \%$ (Figures 5B-D).

To summarize, these results indicate that acute DEHP exposure stimulates excessive ROS production in sperm, as well as trigger altered tyrosine phosphorylation and inhibits the acrosome reaction. Consequently, these changes negatively affect sperm physiology and impact their fertility.

\section{DISCUSSION}

Exposure to EDCs poses a significant risk to reproductive health and fetal development (Hannon et al., 2016; Brehm et al., 2018). In this study, we assessed the effects of acute exposure to four omnipresent EDC's - BPA, DEHP, DMP, and DEP on sperm fertility and early embryo development. While it is well documented that chronic exposure to these compounds can affect both male and female fertility (Latini et al., 2006; vom Saal et al., 2008; Kay et al., 2014; Wu et al., 2014; Patel et al., 2015), the effects of short exposure are less clear. To assess the impact of the chosen EDCs on sperm fertility and preimplantation embryo development, we employed in vitro embryo development and IVF studies. Of all tested EDC's DEHP had the most profound impact on fertilization and sperm fertility. While it is known that chronic exposure to DEHP impairs sperm motility and chromatin DNA stability (Pant et al., 2011; Sumner et al., 2019), its acute effect on sperm fertilizing capacity has not been elucidated. Here, we sought to investigate the impact of 

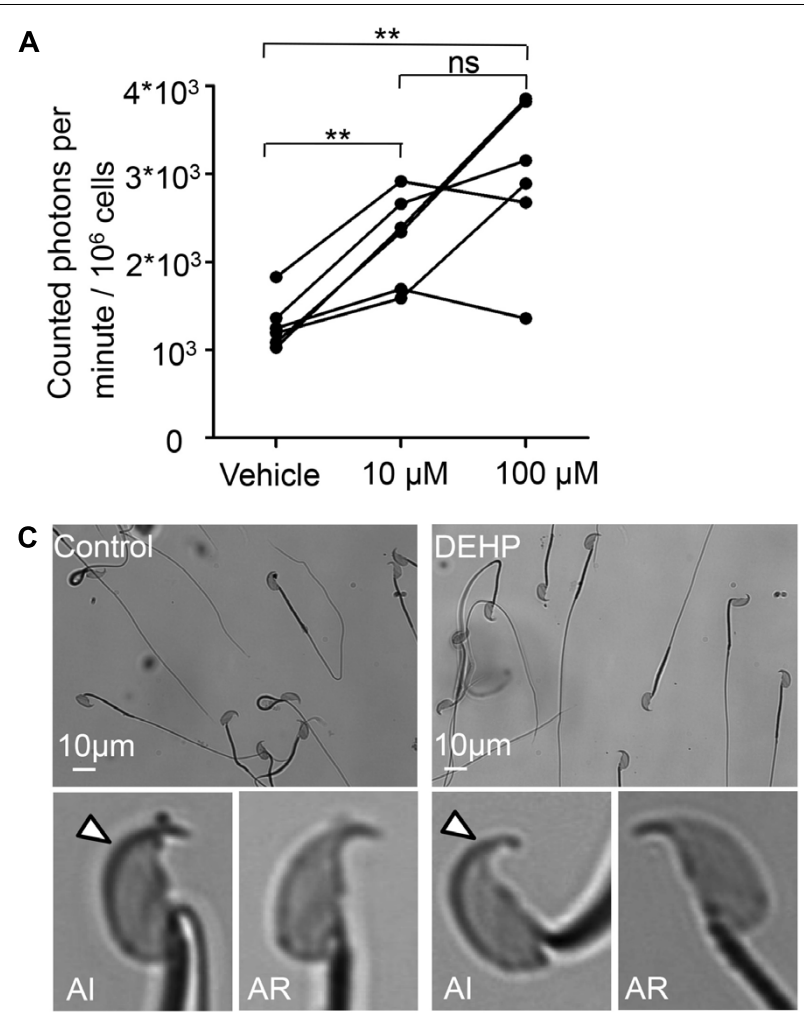
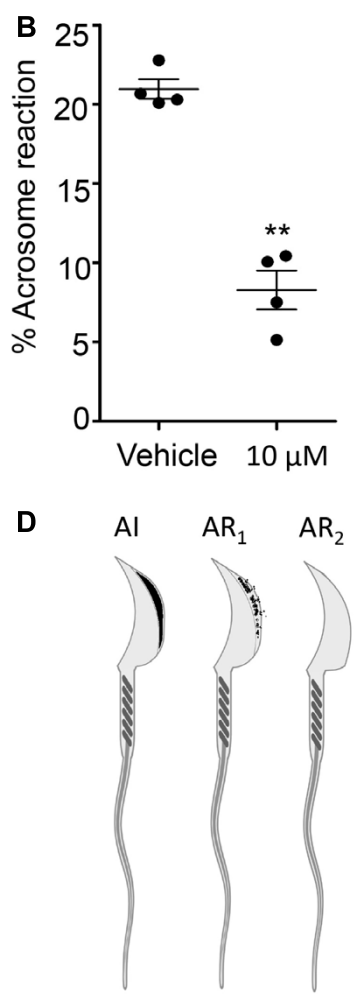

FIGURE 5 | DEHP increases ROS production and decreases spontaneous acrosome reaction in capacitated sperm cells. (A) Luminol-dependent chemiluminescence assay showed increased rates of ROS production in sperm treated with 10 and $100 \mu \mathrm{M}$ DEHP in comparison to the vehicle control $(0 \mu \mathrm{M}$ DEHP). Connected dots represent spermatozoa extracted from the same mouse (a total of six mice were used for this experiment). Each sample was divided into three aliquots and subjected to different conditions. A minimum of $1.35^{\star} 10^{6}$ sperm cells $/ \mathrm{mL}$ was used per condition. (B) Percentage of spontaneously acrosome-reacted spermatozoa subjected to $10 \mu \mathrm{M}$ DEHP or vehicle control $(0.1 \%$ ethanol) during capacitation. Each data point represents the mean of one of four independent experiments. A minimum of 500 cells was evaluated per experiment. (C) Acrosome-reacted (AR) and acrosome-intact (Al) spermatozoa in control and $10 \mu \mathrm{M}$ DEHP. Arrowheads point at intact acrosome caps. "Al" cells have bright blue staining on the dorsal region of the acrosome. "AR" cells have patchy or absent staining. (D) Diagram showing acrosome-reacted (two distinct patterns are indicated as AR1 or partially reacted, and AR2 as fully reacted) and acrosome-intact (Al) spermatozoa. Images were produced using Biorender.com. Data are means \pm S.E.M. ${ }^{* *}$ indicates statistical significance $(P<0.01)$ between control spermatozoa and spermatozoa exposed to $10 \mu \mathrm{M}$ DEHP.

short exposure to DEHP on sperm fertility and the mechanisms by which this EDC exhorts its effects.

Upon absorption, DEHP is distributed throughout the body by the circulatory system. The majority of DEHP is quickly hydrolyzed by the liver into various metabolites, which have been linked to altered fertility and DNA damage in sperm (Hauser et al., 2007). However, a portion of DEHP is stored un-metabolized in the adipose tissue- which acts as a reservoir for lipophilic EDCs (Tanaka et al., 1975; Regnier and Sargis, 2014). Hormonal and neuronal signals regulating the fat tissue can trigger an abrupt release of lipophilic EDCs to the systemic circulation (Regnier and Sargis, 2014). There are two main ways in which sperm can encounter un-metabolized DEHP: via abrupt release from adipose tissue or through release from medical devices, such as a urological catheter- making unmetabolized DEHP a prominent threat to sperm fertility.

Di-2-ethylhexyl phthalate exposure has been previously linked to increased ROS production in somatic cells and oocytes (Ambruosi et al., 2011; Kim et al., 2013; Wu et al., 2014; Tripathi et al., 2019). However, its impact on ROS overproduction in sperm was not elucidated. Here we show that acute exposure to DEHP triggers excessive ROS generation, leading to oxidative damage and ultimately sperm infertility.

While minor ROS generation naturally takes place during early sperm capacitation, this process must be tightly regulated. Mild ROS production triggers an increase in intracellular cAMP, resulting in the activation of Protein Kinase A (PKA). PKA, in turn, carries out a series of controlled tyrosine phosphorylation events in a time-dependent manner (Aitken et al., 1995; Leclerc et al., 1997). Interestingly, certain EDCs such as BPA have been shown to up-regulate PKA's activity leading to an altered phosphorylation pattern downstream of PKA (Rahman et al., 2015). Since mature spermatozoa are transcriptionally and translationally silent cells, post-translational protein modifications such as tyrosine phosphorylation play an essential role in sperm maturation process and their ability to fertilize an egg (Naz and Rajesh, 2004). Unwarranted ROS production leads to over-phosphorylation which significantly alters the maturation process of sperm 
(Villegas et al., 2003; Donà et al., 2011). Moreover, an excess of ROS is cytotoxic to sperm due to their limited antioxidant capacity and their high content of polyunsaturated long-chain fatty acids in the plasma membrane (Jones et al., 1979; Alvarez and Storey, 1982; Aitken and Clarkson, 1987).

Di-2-ethylhexyl phthalate-treated sperm cells had altered capacitation with aberrantly fast tyrosine phosphorylation within the first $60 \mathrm{~min}$ of capacitation. This differs from the gradual increasing protein phosphorylation pattern that was observed in the control condition and previously reported in the literature (Visconti et al., 1995b; Piehler et al., 2006). The detected increase in tyrosine phosphorylation was localized primarily to the mid-piece region of sperm, the flagellar compartment where mitochondria are located. Mitochondrial respiration produces a significant amount of ROS, this process, if unregulated, can damage sperm genomic DNA, lipid and protein structures, and subsequently impair sperm integrity and fertility. Previous reports show that DEHP exposed oocytes and somatic cells produce an excessive amount of ROS via mitochondrial-derived ROS (Rosado-Berrios et al., 2011; Wu et al., 2014; Roth, 2018). However, it has not been shown that DEHP affects spermatozoa in a similar manner. In fact, several EDCs were suggested to affect sperm fertility via CatSper-related mechanism (Schiffer et al., 2014). Here, we show that while murine CatSper was not sensitive to DEHP exposure, this phthalate indeed triggers excessive ROS production and subsequently impairs sperm fertility.

An additional effect of excessive oxidative stress on spermatozoa is lipid peroxidation. Spermatozoa are extremely susceptible to lipid peroxidation due to their high concentration of long- chain polyunsaturated fatty acids (Jones et al., 1979; Alvarez and Storey, 1982; Aitken and Clarkson, 1987; Aitken et al., 2006; Wathes et al., 2007). Alteration of the lipid structure due to peroxidation in sperm leads to a decrease in membrane fluidity (Sikka, 2001; Zalata et al., 2004; Cocuzza et al., 2007; Chen et al., 2013) causing decreased motility and altered acrosome reaction (Aitken and Clarkson, 1987; Griveau et al., 1995; Ichikawa et al., 1999; Zalata et al., 2004). The acrosome reaction is an important step during fertilization in order to expose sperm-egg recognition elements (Inoue et al., 2005). Murine sperm begin to undergo the acrosome reaction in the upper isthmus (La Spina et al., 2016), the part of the oviduct that connects the uterine with the ampulla. Thus, mouse fertile sperm are acrosome-reacted prior to reaching the ampulla, the site of fertilization and before encountering the eggs (Jin et al., 2011). In fact, most acrosome-intact spermatozoa are unable to fertilize the egg and swim away from the zona pellucida (Jin et al., 2011). Thus, sperm ability to undergo the AR at the end of capacitation is highly important for sperm fertility (Yanagimachi, 2011). Here we find that DEHP significantly inhibits the acrosome reaction in capacitated sperm. As a result, DEHP-exposed sperm were largely acrosome-intact and therefore unable to fertilize murine eggs. This explains the reduced levels of fertilization that were observed in IVF and the absence of pronuclei formation. These results indicate that, in addition to its chronic impact on reproductive potential, DEHP also imposes acute damage to sperm by affecting its ability to fertilize and thereby represent a risk to male fertility.

\section{MATERIALS AND METHODS}

\section{Animal Care}

C57BL6 mice were purchased from Jackson Laboratory, Bar Harbor, ME, United States, or Harlan Laboratories (Indianapolis, IN, United States). The mice were kept in a room with controlled light (14 h light and $10 \mathrm{~h}$ darkness) and temperature $\left(23 \pm 0.5^{\circ} \mathrm{C}\right) ; 50-60 \%$ humidity. The mice were fed a standard chow diet (PicoLab Rodent diet 20 and LabDiet, 5053) and hyper-chlorinated water ad libitum. BPA, DEHP, DMP, and DEP (Sigma-Aldrich, St. Louis, MO, United States) were dissolved in ethanol (Sigma-Aldrich). Phthalates were used at a final concentration of 1,2 , and $10 \mu \mathrm{M}$. The concentration range of 1-10 $\mu \mathrm{M}$ for tested EDC's was chosen based on previously reported phthalate concentrations linked to female infertility and on studies that evaluated DEHP plasma concentration in patients who undergo hemodialysis (Nassberger et al., 1987; Reddy et al., 2006). Vehicle controls were performed at the highest concentration.

\section{Embryo Collection From Natural Mating}

Four- to-16- week-old female mice were super- ovulated by the standard procedure previously described (Hoogenkamp and Lewing, 1982). Briefly, 5 IU of pregnant mare serum gonadotropin (PMSG; EMD Millipore) were administered via intraperitoneal injection (i.p.) at 14:30. Forty-eight hours later, 5 IU of human chorionic gonadotropin (hCG; EMD Millipore) were injected. At the time of hCG injection, each female mouse was placed in an individual cage with one proven breeder (310 months old). The following morning, female mice were inspected for vaginal plugs. $20 \mathrm{~h}$ after hCG administration, embryos were dissected out from the oviducts. The isolated oocyte-cumulus complexes were placed in pre-warmed $50 \mu \mathrm{L}$ droplet of Hyaluronidase (80 IU/mL) (LifeGlobal) and were gently pipetted up and down repeatedly in a fine glass pipette until the oocytes were partially denuded. The oocytes were then transferred to a pre-warmed M2 media (Zenith Biotech) supplemented with $4 \mathrm{mg} / \mathrm{mL}$ BSA (Sigma-Aldrich) and washed in 4-5 droplets until all the corona cells were removed. Zygotes from each individual mouse were randomly allocated to different culture conditions for $20 \mathrm{~h}$ of incubation. Since the tested EDCs show low water solubility and high oil solubility, the standard culture of embryos under oil could not be employed. Thus, we cultured the embryos in $500 \mu \mathrm{L}$ KSOM (Zenith Biotech) \pm EDCs at different concentrations for $20 \mathrm{~h}$ in 4 -well dishes $\left(\mathrm{Nunc}^{\mathrm{TM}}\right.$, Sigma-Aldrich) without oil. At the end of the $20 \mathrm{~h}$ incubation, the two cell embryos were briefly washed and allocated to culture dishes, containing $10 \mu \mathrm{L}$ droplets of KSOM (Zenith Biotech) supplemented with $1 \mathrm{mg} / \mathrm{mL}$ BSA (Sigma-Aldrich) overlaid with embryo-suitable light mineral oil (Millipore) in $5 \% \mathrm{CO}_{2}$ and $37^{\circ} \mathrm{C}$. Successful development was considered as morula or blastocyst - the final stage of embryonic development before implantation. To calculate the rate of embryo survival, we 
counted the number of embryos that developed to the morula or blastocyst stage and divided this number by the total number of the zygotes that were harvested.

\section{In vitro Fertilization}

To investigate the influence of EDCs exposure on spermatozoa's ability to fertilize eggs, in vitro fertilization (IVF) experiments were conducted. IVF was performed as previously described (Vergara et al., 1997) with a few modifications. Eggs were recovered from 4 to 16 -week-old female mice by superovulation as described above. $13 \mathrm{~h}$ after hCG injection, the female mice were euthanized, and the oviducts were dissected out. The cumulus masses were isolated from the ampulla region of the oviduct, and incubated in HTF medium (Embryomax, Specialty Media, Millipore MR-070-D), $5 \% \mathrm{CO}_{2}, 37^{\circ} \mathrm{C}$ for $30 \mathrm{~min}$ prior to insemination. Sperm was obtained from a mature C57BL male mouse just before egg harvest. Spermatozoa were recovered by removing the caudae epididymis and placing each cauda separately in a petri dish containing pre-warmed HTF with or without EDCs. The tissue was cut five to six times, and sperm was allowed to swim out into the medium for $20-30 \mathrm{~min}$. The cauda was then removed, and the resultant sperm suspension was left for an additional $30-60 \mathrm{~min}$ in the media at $37^{\circ} \mathrm{C}$ in $5 \% \mathrm{CO}_{2}$ to capacitate. Total time of capacitation was 60-90 min.

Four well plates were used for fertilization. Each well was filled with $700 \mu \mathrm{L}$ of HTF. Sperm was added to each well to a final concentration of 210,000 spermatozoa/mL. Subsequently, the cumulus masses were added to the fertilization dish; care was taken to avoid carry-over of excessive amounts of solution to maintain sperm concentrations. Since the cumulus masses were obtained around the time of ovulation, they were highly compact, making it difficult to quantify the exact number of eggs in each mass. To ensure similar numbers of eggs in each tested condition, two-three cumulus masses were added to each fertilization well. Dishes were placed in the incubator and maintained at $37^{\circ} \mathrm{C}$ in $5 \% \mathrm{CO}_{2}$ for $4 \mathrm{~h}$. After that time, eggs were washed to remove excess sperm and then cultured in $10 \mu \mathrm{L}$ droplets of KSOM supplemented with $1 \mathrm{mg} / \mathrm{mL} \mathrm{BSA}$ and overlaid with embryotested light mineral oil in $5 \% \mathrm{CO}_{2}$ and $37^{\circ} \mathrm{C}$. To assess the rates of successful fertilization, the number of morula or blastocyst embryos produced by IVF on day 5 post insemination was counted and then divided by the number of eggs that were initially used for insemination.

\section{Electrophysiology}

Sperm was collected as previously reported (Wennemuth et al., 2003). Giga-ohm seals between the patch pipette and mouse spermatozoa were formed at the cytoplasmic droplet. Seals were formed in HS solution comprising the following (in $\mathrm{mM}$ ): 130 $\mathrm{NaCl}, 5 \mathrm{KCl}, 1 \mathrm{MgSO}_{4}, 2 \mathrm{CaCl}_{2}$, 5 glucose, 1 sodium pyruvate, 10 lactic acid, 20 HEPES, pH 7.4 adjusted with $\mathrm{NaOH}$. Transition into the whole-cell mode was performed by applying short (1 ms) 499-611 mV voltage pulses, combined with light suction. Access resistance was 15-25 M . Cells were stimulated every $5 \mathrm{~s}$. Data were sampled at $2-5 \mathrm{kHz}$ and filtered at $1 \mathrm{kHz}$. Pipettes (15-20 M $\Omega$ ) for whole-cell patch-clamp recordings of monovalent CatSper currents were filled with the following (in
mM): 130 Cs-methanesulfonate, 70 HEPES/MES, 3 EGTA, 2 EDTA, 0.5 Tris- $\mathrm{HCl}, \mathrm{pH} 7.4$ adjusted with $\mathrm{CsOH}$. Bath divalentfree solution for recording of monovalent CatSper currents contained the following (in $\mathrm{mM}$ ): 140 Cs-methanesulfonate, 40 HEPES/MES, 1 EDTA, pH 7.4 adjusted with $\mathrm{CsOH}$. HS solution was used to record baseline current while measuring monovalent CatSper currents. $1 \mu \mathrm{L} / \mathrm{mL}$ EtOH (vehicle control), $10 \mu \mathrm{M}$ or $100 \mu \mathrm{m}$ DEHP were added to the bath solution right before electrophysiology experiments. $\mathrm{CaCl}_{2}$ was added to this solution in accordance with WinMAXC version 2.05 (C. Patton, Stanford University) to obtain the required free $\mathrm{Ca}^{2+}$ concentration.

\section{Capacitation of Spermatozoa in the Presence of $10 \mu \mathrm{M}$ DEHP to Test the Level of Tyrosine Phosphorylation}

Spermatozoa collection and the assessment of protein tyrosine phosphorylation was performed as previously outlined (Visconti et al., 1995a), with a few modifications. Spermatozoa were recovered by removing the cauda epididymis and placing it into a Petri dish containing HTF with either $1 \mu \mathrm{L} / \mathrm{mL}$ ethanol or $10 \mu \mathrm{M}$ DEHP. The tissue was cut five to six times, and sperm was allowed to swim out for $15-20 \mathrm{~min}$ at $37^{\circ} \mathrm{C}$ in $5 \% \mathrm{CO}_{2}$. The cauda was then removed, and spermatozoa suspension was further incubated at $37^{\circ} \mathrm{C}$ in $5 \% \mathrm{CO}_{2}$. Sperm samples were collected after $30,60,90$, and $120 \mathrm{~min}$ of capacitation and placed into a clean tube. After each sample collection, the sample was centrifuged at $21,000 \times g$ for $1 \mathrm{~min}$. The supernatant was discarded, and the cellular pellet was resuspended in $25 \mu \mathrm{L}$ of $2 \times$ Laemmli sample buffer (Bio-Rad) (Laemmli, 1970). The sample was then boiled for $5 \mathrm{~min}$ at $95^{\circ} \mathrm{C}$ and centrifuged at $21,000 \times g$ for $1 \mathrm{~min}$. Supernatants were transferred to clean tubes, $\beta$-mercaptoethanol was added (to a final concentration of $2.5 \%$ ), and the sample was heated again to $95^{\circ} \mathrm{C}$ for $1 \mathrm{~min} .20 \mu \mathrm{L}$ of the total crude cell lysate from each sample was loaded onto a $4-20 \%$ gradient Tris-HCl Criterion SDS-PAGE (Bio-Rad). After transfer to polyvinylidene fluoride membrane, blots were blocked in $0.1 \%$ PBS-Tween20 (Fisher Scientific) with 3\% IgGfree BSA blocking solution for $1 \mathrm{~h}$ and incubated with antiphosphotyrosine antibody, clone 4G10 (Millipore, 05-321) at a dilution of $1: 2000$ in 1\% IgG-free BSA blocking solution overnight at $+4^{\circ} \mathrm{C}$. The membrane was then washed three times in PBST and probed with a secondary horseradish peroxidaseconjugated antibody (Abcam) at a dilution of 1:15,000 in $1 \times$ PBST. After subsequent washing, the membrane was developed with an ECL SuperSignal West Pico kit (Pierce) according to the manufacturer's instructions. After detection, the membrane was stripped and re-probed with mouse tubulin-alpha ab2 (Sigma-Aldrich), 1:5000 dilution. To quantify the global changes in tyrosine phosphorylation, rectangular boxes were drawn around each lane of the western blots' images. Each lane's optical density was normalized. First, the detected signals were normalized to the loading control, acetylated tubulin. Subsequently, each lane was normalized to the control lane at $120 \mathrm{~min}$ of capacitation. 


\section{Spontaneous Acrosome Reaction in the Presence of $10 \mu$ M DEHP in Capacitated Spermatozoa}

Assessment of the acrosomal status was done as previously reported (Larson and Miller, 1999) with a few adjustments. In summary, the right and left caudae epididymides were surgically removed. One cauda was placed in HTF medium supplemented with $1 \mu \mathrm{l} / \mathrm{mL}$ ethanol while the second one was placed in HTF medium containing $10 \mu \mathrm{M}$ DEHP. Each cauda was cut five to six times, and sperm were allowed to swim out for 15-20 min at $37^{\circ} \mathrm{C}$ in $5 \% \mathrm{CO}_{2}$. The cauda was then removed, and the resultant spermatozoa suspension was left to capacitate for $60 \mathrm{~min}$. On average, the concentration of sperm in each condition was 2$5 \times 10$ cells $/ \mathrm{mL}$. After $60 \mathrm{~min}$ of capacitation, spermatozoa suspension was transferred to clean microtubes and centrifuged at $300 \times g$ for $5 \mathrm{~min}$ at room temperature. The cells were then fixed in $4 \%$ PFA in $1 \mathrm{X}$ PBS for $15 \mathrm{~min}$. At the end of fixation, an equal volume of $0.1 \mathrm{M}$ ammonium acetate was added. The microfuges were centrifuged at $800 \times g$ for $5 \mathrm{~min}$. The supernatant was removed, and sperm cells were resuspended in the remaining $100 \mu \mathrm{L} .30 \mu \mathrm{L}$ of sperm suspension was spotted onto non-charged microscope slides and gently spread out with a glass pipette. The samples were allowed to air-dry for $15 \mathrm{~min}$. Subsequently, the slides were washed in Milli-Q water followed by a methanol wash, and then Milli-Q water again, each wash step was done for $5 \mathrm{~min}$. The slides were subsequently submerged in Coomassie brilliant blue (Sigma-Aldrich) solution for $2 \mathrm{~min}$ (0.11 g Coomassie brilliant blue, $20 \mathrm{~mL}$ water, $25 \mathrm{~mL}$ Methanol, and $5 \mathrm{~mL}$ glacial acetic acid). Next, the slides were rinsed in Milli$\mathrm{Q}$ water to remove excess Coomassie and mounted with Mowiol mounting medium (Millipore). After the cells were mounted, the acrosomal status was immediately assessed to avoid diffusion of the stain. Acrosome intact cells had bright blue staining on the dorsal region of the acrosome. Acrosome reacted cells had patchy or absent staining. 500 sperm cells per condition (100-200 cells per slide, 3-5 slides per condition) were assessed.

\section{Flow Cytometry}

Spermatozoa was capacitated in the presence of either $10 \mu \mathrm{M}$ DEHP or vehicle control. To detect phosphotyrosine residues in capacitated sperm by flow cytometry we followed the methodology previously outlined (Barbonetti et al., 2008), with few modifications. Sperm aliquots $\left(3 \times 10^{6}\right)$ were taken at $60 \mathrm{~min}$ of capacitation and fixed in $3.7 \%$ PFA in $1 \times$ PBS for $10 \mathrm{~min}$ at room temperature. To remove the PFA, the cells were centrifuged at $500 \times g$ for $10 \mathrm{~min}$. The supernatant was removed, and the cellular pellet was resuspended in $1 \times$ PBS. The cells were washed twice. Next, the cells were permeabilized in $0.1 \%$ Triton X-100 for $10 \mathrm{~min}$ at RT. Non-specific binding sites were blocked by $0.1 \%$ BSA in PBST for $30 \mathrm{~min}$ at RT. To detect phosphotyrosine, we conjugated an anti-phosphotyrosine antibody clone 4G10 with a CF 647 dye (Mix-n-Stain ${ }^{\text {TM }}$ Antibody Labeling Kit, Biotium), as per the manufacturer's instructions. To label the cells, sperm were incubated with $10 \mu \mathrm{g} / \mathrm{ml}$ of the conjugated antibody in PBS with $0.1 \%$ BSA for $1 \mathrm{~h}$ at RT. Labeled spermatozoa were then washed in PBS and resuspended in $250 \mu \mathrm{L}$ PBS for flow cytometric analysis.
10,000 cells per sample were analyzed. Sperm fluorescence was quantified using the BD LSR Fortessa flow cytometer equipped with an argon laser tuned far red spectrum. flow cytometry analysis was performed with the aid of a positive and a negative control for each experiment. FlowJo ${ }^{\mathrm{TM}}$ Software was used for data analysis. The region of interest was selected based on sperm forward scatter (FSC, relative cell size) and side scatter (SSC, cell internal complexity) to eliminate cellular debris.

\section{ROS Production Detection by Chemiluminescence Assay}

Spermatozoa were recovered by removing both caudae epididymides and placing them into a $30 \mathrm{~mm}$ Petri dish containing HS media. Cells were allowed to swim out for 15$20 \mathrm{~min}$. Subsequently, the sperm suspension was equally divided between three Eppendorf tubes and spun down at $300 \times g$ for $7 \mathrm{~min}$. After the removal of the supernatant, the cells were resuspended in an equal volume of HTF containing either ethanol or DEHP. The control tube contained $1 \mu \mathrm{L} / \mathrm{mL}$ ethanol and the treatment tube contained either $10 \mu \mathrm{M}$ or a $100 \mu \mathrm{M}$ DEHP. The suspensions were then capacitated at $37^{\circ} \mathrm{C}$ in $5 \% \mathrm{CO}_{2}$ for $60 \mathrm{~min}$. Detection of reactive oxygen species generated by sperm cells was done using the chemiluminescent agent - luminol following a previously described procedure (Saleh and Agarwal, 2002; Agarwal et al., 2008). The chemiluminescent probe, luminol (Sigma-Aldrich, A8511-5G.) was freshly prepared before each experiment. After $60 \mathrm{~min}$ of capacitation, the samples were spun down at $300 \times g$ for $7 \mathrm{~min}$ and resuspended in $125 \mu \mathrm{M}$ luminol in DPBS. Negative control, test sample, and positive control were prepared. $100 \mu \mathrm{L}$ of $30 \%$ hydrogen peroxide solution was added to the positive control. A $100 \mu \mathrm{L}$ aliquot of the cell suspension was taken from each sample for sperm count. The samples were then taken for Chemiluminescence measurements using the Lumicycle 32 (Actimetrics, Inc., Wilmette, IL, United States). The luminometer measured Chemiluminescence at $37^{\circ} \mathrm{C}$ for 5 min. ROS production was expressed as counted photons per minute $(\mathrm{CPM}) / 10^{6}$ sperm. Data were recorded using Actimetrics Lumicycle Data Collection software and analyzed using the Actimetrics Lumicycle Analysis program.

\section{Statistical Analyses}

For statistical analyses used in the manuscript the GraphPad Prism 5 software (GraphPad Software, Inc., La Jolla, CA, United States) was used. Unpaired $t$-test was used to determine statistical significance for embryo survival, IVF and Chemiluminescence experiments, and assigning $p \leq 0.05$ as the limit. Paired $t$-test was used for the AR, PY and flow cytometry experiments. All results are shown with the standard error of the mean. The significance of changes are indicated as follows: ${ }^{*} p \leq 0.05,{ }^{* *} p \leq 0.01,{ }^{* * *} p \leq 0.001$.

\section{DATA AVAILABILITY STATEMENT}

All datasets generated for this study are included in the article/Supplementary Material. 


\section{ETHICS STATEMENT}

All experiments were performed in accordance with $\mathrm{NIH}$ guidelines for animal research and approved by UC Berkeley Animal Care and Use Committee (AUP 2015-07-7742), with every effort made to minimize suffering for the animals.

\section{AUTHOR CONTRIBUTIONS}

LGK and PL conceived the project, designed the experiments, and wrote the manuscript. LGK performed all studies, data acquisition, and analysis for the manuscript. JD helped with acrosome reaction, tyrosine phosphorylation, chemiluminescence, and flow cytometry studies. NP assisted with flow cytometry experiments. JM and LJK helped with lumicycle and data analysis of luminol studies. All authors discussed the results and commented on the manuscript.

\section{FUNDING}

This work was supported by March of Dimes Basil O'Connor Starter Scholar Research Award \#5FY13-204, Pew Biomedical Scholars Award, Chau Hoi Shuen Foundation Women in Science Program Award, and Packer Wentz Endowment Will (to PL), as well as Male Contraceptive Initiative grant to LGK and Summer Research Fellowship to JD.

\section{ACKNOWLEDGMENTS}

We thank Dr. Andrew Modzelewski for his valuable advice and technical assistance with embryo work, and Monika Haoui for the help with manuscript editing.

\section{SUPPLEMENTARY MATERIAL}

The Supplementary Material for this article can be found online at: https://www.frontiersin.org/articles/10.3389/fcell.2020.00426/ full\#supplementary-material

FIGURE S1 | Murine embryo survival post exposure to $2 \mu \mathrm{M}$ of tested EDCs. In vitro embryo development on day 5 post fertilization. (A) Representative images of blastocysts previously exposed at the zygote stage to $2 \mu \mathrm{M}$ DEP for $20 \mathrm{~h}$. (B) Representative images of blastocysts previously exposed at the zygote stage to $2 \mu \mathrm{M}$ DMP for $20 \mathrm{~h}$. Scale bars are $50 \mu \mathrm{m}$.

FIGURE S2 | Fertilization rate of murine eggs exposed to spermatozoa treated with $2 \mu \mathrm{M}$ of tested EDCs. (A) Representative images of blastocysts obtained after murine eggs were introduced to sperm previously exposed $2 \mu \mathrm{M}$ DEP. (B) Representative images of blastocysts obtained after murine eggs were introduced to sperm previously exposed $2 \mu \mathrm{M}$ DMP. (C) Representative images of blastocysts obtained after murine eggs were introduced to sperm previously exposed $2 \mu \mathrm{M}$ BPA. Scale bars are $50 \mu \mathrm{m}$.

FIGURE S3 | Murine CatSper is not affected by DEHP. (A) Representative monovalent whole-cell CatSper currents (ICatSper) recorded from a murine spermatozoon in the absence (black) and presence of $10 \mu \mathrm{M}$ (blue) and $100 \mu \mathrm{M}$ DEHP (red). ICatSper were activated by a voltage ramp from -80 to $+80 \mathrm{mV}$ from a holding potential of $0 \mathrm{mV}$. Voltage protocol is shown above the currents. The panel on the right shows the main conducting ion of the pipette and bath solutions. (B) Averaged $I_{\text {CatSper }}$ densities recorded from murine epididymal spermatozoa in the absence and presence of DEHP. Data are means \pm S.E.M. An average of 3 independent experiments is shown.

FIGURE S4 | Representative dot plot of side (SSC-A) versus forward (FSC-A) scatter showing flow-cytometry data obtained for sperm. (A) The region of interest demarcated by solid lines was selected to eliminate cellular debris. (B-F) Representative flow cytometry histograms from five independent experiments. Mean fluorescence intensities (MFI) normalized to mode show an increase in global tyrosine phosphorylation in $10 \mu \mathrm{M}$ DEHP treated spermatozoa (red) at 60 min of capacitation compared to the vehicle control (blue). The background fluorescence detected in unstained spermatozoa is shown in gray. Tyrosine phosphoproteins were detected using a CF 647 dye conjugated to anti-PY (monoclonal antibody).

FIGURE S5 | Time course of capacitation-associated tyrosine phosphorylation of specific sperm proteins is altered by exposure to DEHP. Levels of relative tyrosine phosphorylation obtained from each of the four protein bands at the corresponding molecular weights: 75, 95, 170, and $270 \mathrm{kDa}$. (A) The density of the $75 \mathrm{kDa}$ protein band was normalized to the densities of the loading control, followed by normalization to the control at $120 \mathrm{~min}$. Each data point represents the average of one of the three independent experiments. (B) The $95 \mathrm{kDa}$ protein band normalized as in (A). (C) The $170 \mathrm{kDa}$ protein band normalized as in (A). (D) The $270 \mathrm{kDa}$ protein band normalized as in (A). Normalization to the control band at 120 min was chosen as the strongest physiological phosphorylation signal.

TABLE S1 | (A-D) Murine embryo development after $20 \mathrm{~h}$ exposure to DMP, BPA, DEP, or DEHP. Embryo development was assessed on day 5 post fertilization. The column "progression to blastocyst stage per experiment, \%" represents the percentage of embryos that reached blastocyst or morula stage. This number was calculated by dividing the number of all embryos that reached blastocyst or morula stage to the number of all collected zygotes per each experiment. Zygotes were obtained from naturally mated super-ovulated females. Each condition was assessed by 3-8 independent experiments. (A) Embryo development after $20 \mathrm{~h}$ exposure to DMP at $0,1,2$, and $10 \mu \mathrm{M}$ and subsequent embryo culture in DMP-free media. (B) Embryo development after $20 \mathrm{~h}$ exposure to the indicated concentration of BPA and subsequent culture in BPA-free media. (C) Embryo development after $20 \mathrm{~h}$ exposure to the indicated concentration of DEP and subsequent culture in DEP-free media. (D) Embryo development after $20 \mathrm{~h}$ exposure to the indicated concentration of DEHP and subsequent culture in DEHP-free media.

TABLE S2 | The development of murine zygotes isolated from naturally mated super-ovulated females and after their exposure to $0.1 \%$ ethanol for $20 \mathrm{~h}$ in the culture media. Embryo development was assessed on day 5 post fertilization and represents the percentage of embryos that reached blastocyst or morula stage. This number was calculated by dividing the number of all embryos that reached blastocyst or morula stage by the number of all collected zygotes per experiment.

TABLE S3 | (A-D) Development of in vitro fertilized mouse embryos obtained after murine eggs were introduced to the sperm previously exposed to DMP, BPA, DEP, or DEHP for 60-90 min. Embryo development was assessed on day 5 post fertilization. The "Progression to the blastocyst stage per experiment, \%" column represents the percentage of embryos that reached blastocyst or morula stage. This number was calculated by dividing the number of all embryos that reached blastocyst or morula stage to the number of all collected and inseminated eggs per each experiment. Each condition was assessed by 3-5 independent experiments. (A) In vitro embryo development after eggs insemination with 0, 1, 2, or $10 \mu \mathrm{M}$ DMP-treated sperm (B) In vitro embryo development after eggs were inseminated with spermatozoa previously exposed to the indicated concentration of BPA. (C) In vitro embryo development after eggs were inseminated with the spermatozoa treated with corresponding concentrations of DEP. (D) In vitro embryo development after murine eggs were inseminated with spermatozoa treated with corresponding concentrations of DEHP.

TABLE S4 | Development of embryos derived from the murine eggs that were subjected to in vitro fertilization (IVF) with murine sperm previously exposed to $0.1 \%$ ethanol for 60-90 min. Embryo development was assessed on the day 5 post IVF and represents the percentage of embryos that reached blastocyst or morula stage. This number was calculated by dividing the number of all embryos 
that reached blastocyst or morula stage to the number of all collected eggs per each independent experiment.

TABLE S5 | Effect of $10 \mu \mathrm{M}$ DEHP on fertilization and pronuclei formation. Pronucleus (PN) formation was assessed $9 \mathrm{~h}$ after egg insemination with sperm cells previously exposed to $10 \mu \mathrm{M}$ DEHP. Three independent experiments were carried out.

TABLE S6 | Assessment of the global tyrosine phosphorylation by flow cytometry. Tyrosine phosphoproteins were detected by flow cytometry using a CF 647 dye conjugated to anti-PY antibody. Five independent experiments were carried out. Sperm concentrations were normalized between all conditions for each experiment. Values are mean fluorescence intensity (MFI) detected in the

\section{REFERENCES}

Agarwal, A., Cocuzza, M., Abdelrazik, H., and Sharma, R. K. (2008). Oxidative Stress Measurement in Patients with Male or Female Factor Infertility. Trivandrum: Transworld Research Network.

Aitken, R. J., and Clarkson, J. S. (1987). Cellular basis of defective sperm function and its association with the genesis of reactive oxygen species by human spermatozoa. J. Reprod. Fertil. 81, 459-469. doi: 10.1530/jrf.0.081 0459

Aitken, R. J., Paterson, M., Fisher, H., Buckingham, D. W., and Van Duin, M. (1995). Redox regulation of tyrosine phosphorylation in human spermatozoa and its role in the control of human sperm function. J. Cell Sci. 108, 2017-2025.

Aitken, R. J., Wingate, J. K., De Iuliis, G. N., Koppers, A. J., and Mclaughlin, E. A. (2006). Cis-unsaturated fatty acids stimulate reactive oxygen species generation and lipid peroxidation in human spermatozoa. J Clin Endocrinol Metab 91, 4154-4163. doi: 10.1210/jc.2006-1309

Alvarez, J. G., and Storey, B. T. (1982). Spontaneous lipid peroxidation in rabbit epididymal spermatozoa: its effect on sperm motility. Biol. Reprod. 27, 11021108. doi: 10.1095/biolreprod27.5.1102

Alvau, A., Battistone, M. A., Gervasi, M. G., Navarrete, F. A., Xu, X., SánchezCárdenas, C., et al. (2016). The tyrosine kinase FER is responsible for the capacitationassociated increase in tyrosine phosphorylation in murine sperm. Development 143, 2325-2333. doi: 10.1242/dev.136499

Ambruosi, B., Filioli Uranio, M., Sardanelli, A. M., Pocar, P., Martino, N. A., Paternoster, M. S., et al. (2011). Correction: in vitro acute exposure to DEHP affects oocyte meiotic maturation, energy and oxidative stress parameters in a large animal model. PLoS One 6:e27452. doi: 10.1371/journal.pone.0027452

Api, A. M. (2001). Toxicological profile of diethyl phthalate: a vehicle for fragrance and cosmetic ingredients. Food Chem. Toxicol. 39, 97-108. doi: 10.1016/S02786915(00)00124-1

Austin, C. R. (1951). Observations on the penetration of the sperm in the mammalian egg. Aust. J. Sci. Res. B 4, 581-596. doi: 10.1071/bi9510581

Avella, M. A., and Dean, J. (2011). Fertilization with acrosome-reacted mouse sperm: implications for the site of exocytosis. Proc. Natl. Acad. Sci. U.S.A. 108, 19843-19844. doi: 10.1073/pnas.1118234109

Barbonetti, A., Vassallo, M. R. C., Cinque, B., Antonangelo, C., Sciarretta, F., Santucci, R., et al. (2008). Dynamics of the global tyrosine phosphorylation during capacitation and acquisition of the ability to fuse with oocytes in human spermatozoa1. Biol. Reprod. 79, 649-656. doi: 10.1095/biolreprod.108.06 999.00145.x8254

Bloom, M. S., Whitcomb, B. W., Chen, Z., Ye, A., Kannan, K., and Buck Louis, G. M. (2015). Associations between urinary phthalate concentrations and semen quality parameters in a general population. Hum. Reprod. 30, 2645-2657. doi: 10.1093/humrep/dev219

Brehm, E., and Flaws, J. A. (2019). Transgenerational effects of endocrinedisrupting chemicals on Male and female reproduction. Endocrinology 160, 1421-1435. doi: 10.1210/en.2019-00034

Brehm, E., Rattan, S., Gao, L., and Flaws, J. A. (2018). Prenatal exposure to Di(2Ethylhexyl) phthalate causes long-term transgenerational effects on female reproduction in mice. Endocrinology 159, 795-809. doi: 10.1210/en.2017-03004

Burdorf, A., Brand, T., Jaddoe, V. W., Hofman, A., Mackenbach, J. P., and Steegers, E. A. P. (2011). The effects of work-related maternal risk factors on time to pregnancy, preterm birth and birth weight: the seneration R study. Occup. Environ. Med. 68, 197-204. doi: 10.1136/oem.2009.046516
APC-Cy7-A channel normalized to mode. A ratio of the normalized detected MFI in the $10 \mu \mathrm{M}$ DEHP-treated sperm cells vs. the vehicle control was used to calculate the fold increase in global fluorescence.

TABLE S7 | Detection of reactive oxygen species (ROS) in murine spermatozoa treated with DEHP compared with vehicle control. The luminol-dependent chemiluminescence assay was used to detect ROS production. Six independent experiments were carried out. A minimum of $1.35^{*} 10^{6}$ sperm cells/mL were used per condition. ROS production was expressed as counted photons per minute (CPM) $/ 10^{6}$. Each row represents an individual experiment. For each experiment, sperm concentrations were normalized between all conditions.

Calafat, A. M., Kuklenyik, Z., Reidy, J. A., Caudill, S. P., Ekong, J., and Needham, L. L. (2005). Urinary concentrations of bisphenol A and 4-Nonylphenol in a human reference population. Environ. Health Perspect. 113, 391-395. doi: 10.1289/ehp.7534

Carlson, A. E., Westenbroek, R. E., Quill, T., Ren, D., Clapham, D. E., Hille, B., et al. (2003). CatSper1 required for evoked Ca2+ entry and control of flagellar function in sperm. Proc. Natl. Acad. Sci. U.S.A. 100, 14864-14868. doi: $10.1073 /$ pnas. 2536658100

Chang, M. C. (1957). A detrimental effect of seminal plasma on the fertilizing capacity of sperm. Nature 179, 258-259. doi: 10.1038/179258a0

Chen, S. J., Allam, J. P., Duan, Y. G., and Haidl, G. (2013). Influence of reactive oxygen species on human sperm functions and fertilizing capacity including therapeutical approaches. Arch. Gynecol. Obstet. 288, 191-199. doi: 10.1007/ s00404-013-2801-4

Cocuzza, M., Sikka, S. C., Athayde, K. S., and Agarwal, A. (2007). Clinical relevance of oxidative stress and sperm chromation damage in male infertility: an evidence based analysis. Int. Braz. J. Urol. 33, 603-621. doi: 10.1590/S167755382007000500002

Davis, B. K. (1981). Timing of fertilization in mammals: sperm cholesterol/phospholipid ratio as a determinant of the capacitation interval. Proc. Natl. Acad. Sci. U.S.A. 78, 7560-7564. doi: 10.1073/pnas.78.12.7560

Donà, G., Fiore, C., Andrisani, A., Ambrosini, G., Brunati, A., Ragazzi, E., et al. (2011). Evaluation of correct endogenous reactive oxygen species content for human sperm capacitation and involvement of the NADPH oxidase system. Hum. Reprod. 26, 3264-3273. doi: 10.1093/humrep/der321

Duty, S. M., Silva, M. J., Barr, D. B., Brock, J. W., Ryan, L., Chen, Z., et al. (2003). Phthalate exposure and human semen parameters. Epidemiology 14, 269-277. doi: 10.1097/01.ede.0000059950.11836.16

Fernandez, M. F., Arrebola, J. P., Taoufiki, J., Navalón, A., Ballesteros, O., Pulgar, R., et al. (2007). Bisphenol-A and chlorinated derivatives in adipose tissue of women. Reprod. Toxicol. 24, 259-264. doi: 10.1016/j.reprotox.2007.06.007

Fujii, S., Yabe, K., Furukawa, M., Hirata, M., Kiguchi, M., and Ikka, T. (2005). A two-generation reproductive toxicity study of diethyl phthalate (DEP) in rats. J. Toxicol. Sci. 30, S97-S116. doi: 10.2131/jts.30.S97

Green, R., Hauser, R., Calafat, A. M., Weuve, J., Schettler, T., Ringer, S., et al. (2005). Use of di(2-ethylhexyl) phthalate-containing medical products and urinary levels of mono(2-ethylhexyl) phthalate in neonatal intensive care unit infants. Environ. Health Perspect. 113, 1222-1225. doi: 10.1289/ehp.7932

Griveau, J. F., Renard, P., and Le Lannou, D. (1995). Superoxide anion production by human spermatozoa as a part of the ionophore-induced acrosome reaction process. Int. J. Androl. 18, 67-74. doi: 10.1111/j.1365-2605.1995.tb0 0388.x

Hannon, P. R., Niermann, S., and Flaws, J. A. (2016). Acute exposure to Di(2Ethylhexyl) phthalate in adulthood causes adverse reproductive outcomes later in life and accelerates reproductive aging in female mice. Toxicol. Sci. 150, 97-108. doi: 10.1093/toxsci/kfv317

Hauser, R., and Calafat, A. M. (2005). Phthalates and human health. Occup. Environ. Med. 62, 806-818. doi: 10.1136/oem.2004.017590

Hauser, R., Meeker, J. D., Duty, S., Silva, M. J., and Calafat, A. M. (2006). Altered semen quality in relation to urinary concentrations of phthalate monoester and oxidative metabolites. Epidemiology 17, 682-691. doi: 10.1097/01.ede. 0000235996.89953.d7

Hauser, R., Meeker, J. D., Singh, N. P., Silva, M. J., Ryan, L., Duty, S., et al. (2007). DNA damage in human sperm is related to urinary levels of phthalate 
monoester and oxidative metabolites. Hum. Reprod. 22, 688-695. doi: 10.1093/ humrep/del428

Hoogenkamp, H., and Lewing, P. (1982). Superovulation in mice in relation to their age. Vet. Q. 4, 47-48. doi: 10.1080/01652176.1982.9693838

Huang, Y., Wu, C., Ye, Y., Zeng, J., Zhu, J., Li, Y., et al. (2019). The increase of ROS caused by the interference of DEHP with JNK/p38/p53 pathway as the reason for hepatotoxicity. Int. J. Environ. Res. Public Health 16:356. doi: 10.3390/ijerph16030356

Hunt, P. A., Susiarjo, M., Rubio, C., and Hassold, T. J. (2009). The bisphenol a experience: a primer for the analysis of environmental effects on mammalian reproduction1. Biol. Reprod. 81, 807-813. doi: 10.1095/biolreprod.109.077008

Ichikawa, T., Oeda, T., Ohmori, H., and Schill, W. B. (1999). Reactive oxygen species influence the acrosome reaction but not acrosin activity in human spermatozoa. Int. J. Androl. 22, 37-42. doi: 10.1046/j.1365-2605.1999.00 145. $\mathrm{x}$

Inoue, N., Ikawa, M., Isotani, A., and Okabe, M. (2005). The immunoglobulin superfamily protein Izumo is required for sperm to fuse with eggs. Nature 434, 234-238. doi: 10.1038/nature03362

Jin, M., Fujiwara, E., Kakiuchi, Y., Okabe, M., Satouh, Y., Baba, S. A., et al. (2011). Most fertilizing mouse spermatozoa begin their acrosome reaction before contact with the zona pellucida during in vitro fertilization. Proc. Natl. Acad. Sci. U.S.A. 108, 4892-4896. doi: 10.1073/pnas.1018202108

Jones, R., Mann, T., and Sherins, R. (1979). Peroxidative breakdown of phospholipids in human spermatozoa, spermicidal properties of fatty acid peroxides, and protective action of seminal plasma. Fertil. Steril. 31, 531-537. doi: 10.1016/S0015-0282(16)43999-3

Kasahara, E., Sato, E. F., Miyoshi, M., Konaka, R., Hiramoto, K., Sasaki, J., et al. (2002). Role of oxidative stress in germ cell apoptosis induced by di(2-ethylhexyl)phthalate. Biochem. J. 365, 849-856. doi: 10.1042/BJ2002 0254

Kavlock, R., Boekelheide, K., Chapin, R., Cunningham, M., Faustman, E., Foster, P., et al. (2002). NTP center for the evaluation of risks to human reproduction: phthalates expert panel report on the reproductive and developmental toxicity of di(2-ethylhexyl) phthalate. Reprod. Toxicol. 16, 529-653. doi: 10.1016/S08906238(02)00032-1

Kay, V. R., Bloom, M. S., and Foster, W. G. (2014). Reproductive and developmental effects of phthalate diesters in males. Crit. Rev. Toxicol. 44, 467-498. doi: 10.3109/10408444.2013.875983

Kim, J. H., Park, H. Y., Bae, S., Lim, Y.-H., and Hong, Y.-C. (2013). Diethylhexyl phthalates is associated with insulin resistance via oxidative stress in the elderly: a panel study. PLoS One 8:e71392. doi: 10.1371/journal.pone.007 1392

Kirichok, Y., Navarro, B., and Clapham, D. E. (2006). Whole-cell patch-clamp measurements of spermatozoa reveal an alkaline-activated $\mathrm{Ca} 2+$ channel. Nature 439, 737-740. doi: 10.1038/nature04417

La Spina, F. A., Puga Molina, L. C., Romarowski, A., Vitale, A. M., Falzone, T. L., Krapf, D., et al. (2016). Mouse sperm begin to undergo acrosomal exocytosis in the upper isthmus of the oviduct. Dev. Biol. 411, 172-182. doi: 10.1016/j.ydbio. 2016.02.006

Laemmli, U. K. (1970). Cleavage of structural proteins during the assembly of the head of bacteriophage T4. Nature 227, 680-685. doi: 10.1038/227680a0

Larson, J. L., and Miller, D. J. (1999). Simple histochemical stain for acrosomes on sperm from several species. Mol. Reprod. Dev. 52, 445-449. doi: 10.1002/(sici) 1098-2795(199904)52:4<445::aid-mrd14>3.0.co;2-6

Latini, G., Del Vecchio, A., Massaro, M., Verrotti, A., and De Felice, C. (2006). Phthalate exposure and male infertility. Toxicology 226, 90-98. doi: 10.1016/j. tox.2006.07.011

Leclerc, P., De Lamirande, E., and Gagnon, C. (1997). Regulation of proteintyrosine phosphorylation and human sperm capacitation by reactive oxygen derivatives. Free Radic. Biol. Med. 22, 643-656. doi: 10.1016/S0891-5849(96) 00379-6

Lovekamp, T. N., and Davis, B. J. (2001). Mono-(2-ethylhexyl) phthalate suppresses aromatase transcript levels and estradiol production in cultured rat granulosa cells. Toxicol. Appl. Pharmacol. 172, 217-224. doi: 10.1006/taap.2001.9156

Meeker, J. D., Calafat, A. M., and Hauser, R. (2009). Urinary metabolites of di(2ethylhexyl) phthalate are associated with decreased steroid hormone levels in adult men. J. Androl. 30, 287-297. doi: 10.2164/jandrol.108.006403
Mei, Y., Rongshuang, M., Ruizhi, Z., Hongyuan, H., Qiyue, T., and Shu-hua, Z. (2019). Effects of dimethyl phthalate (DMP) on serum sex hormone levels and apoptosis in C57 female mice. Int. J. Endocrinol. Metab. $17: e 82882$.

Muncke, J. (2011). Endocrine disrupting chemicals and other substances of concern in food contact materials: an updated review of exposure, effect and risk assessment. J. Steroid Biochem. Mol. Biol. 127, 118-127. doi: 10.1016/j.jsbmb. 2010.10.004

Nassar, A., Mahony, M., Morshedi, M., Lin, M. H., Srisombut, C., and Oehninger, S. (1999). Modulation of sperm tail protein tyrosine phosphorylation by pentoxifylline and its correlation with hyperactivated motility. Fertil. Steril. 71, 919-923. doi: 10.1016/S0015-0282(99)00013-8

Nassberger, L., Arbin, A., and Ostelius, J. (1987). Exposure of patients to phthalates from polyvinyl chloride tubes and bags during dialysis. Nephron 45, 286-290. doi: 10.1159/000184165

Naz, R. K., and Rajesh, P. B. (2004). Role of tyrosine phosphorylation in sperm capacitation / acrosome reaction. Reprod. Biol. Endocrinol. 2:75. doi: 10.1186/ 1477-7827-2-75

Ochsendorf, F. R., Thiele, J., Fuchs, J., Schüttau, H., Freisleben, H. J., Buslau, M., et al. (1994). Chemiluminescence in semen of infertile men. Andrologia 26, 289-293. doi: 10.1111/j.1439-0272.1994.tb00804.x

Pan, G., Hanaoka, T., Yu, L., Na, J., Yamano, Y., Hara, K., et al. (2011). Associations between hazard indices of di-n-butylphthalateand di-2-ethylhexylphthalate exposure and serum reproductive hormone levels among occupationally exposed and unexposed Chinese men. Int. J. Androl. 34, e397-e406. doi: 10. 1111/j.1365-2605.2011.01201.x

Pant, N., Pant, A., Shukla, M., Mathur, N., Gupta, Y., and Saxena, D. (2011). Environmental and experimental exposure of phthalate esters: the toxicological consequence on human sperm. Hum. Exp. Toxicol. 30, 507-514. doi: 10.1177/ 0960327110374205

Patel, S., Zhou, C., Rattan, S., and Flaws, J. A. (2015). Effects of endocrinedisrupting chemicals on the Ovary1. Biol. Reprod. 93, 1-9. doi: 10.1095/ biolreprod.115.130336

Pearson, S. D., and Trissel, L. A. (1993). Leaching of diethylhexyl phthalate from polyvinyl chloride containers by selected drugs and formulation components. Am. J. Hosp. Pharm. 50, 1405-1409. doi: 10.1093/ajhp/50.7.1405

Piehler, E., Petrunkina, A. M., Ekhlasi-Hundrieser, M., and Töpfer-Petersen, E. (2006). Dynamic quantification of the tyrosine phosphorylation of the sperm surface proteins during capacitation. Cytometry. A 69, 1062-1070. doi: 10.1002/ cyto.a. 20338

Qi, H., Moran, M. M., Navarro, B., Chong, J. A., Krapivinsky, G., Krapivinsky, L., et al. (2007). All four CatSper ion channel proteins are required for male fertility and sperm cell hyperactivated motility. Proc. Natl. Acad. Sci. U.S.A. 104, 1219-1223. doi: 10.1073/pnas.0610286104

Rahman, M. S., Kwon, W.-S., Lee, J.-S., Yoon, S.-J., Ryu, B.-Y., and Pang, M.G. (2015). Bisphenol-A affects male fertility via fertility-related proteins in spermatozoa. Sci. Rep. 5:9169. doi: 10.1038/srep09169

Reddy, B. S., Rozati, R., Reddy, B. V. R., and Raman, N. V. V. S. S. (2006). Association of phthalate esters with endometriosis in Indian women. BJOG 113, 515-520. doi: 10.1111/j.1471-0528.2006.00925.x

Regnier, S. M., and Sargis, R. M. (2014). Adipocytes under assault: environmental disruption of adipose physiology. Biochim. Biophys. Acta 1842, 520-533. doi: 10.1016/j.bbadis.2013.05.028

Ren, D., Navarro, B., Perez, G., Jackson, A. C., Hsu, S., Shi, Q., et al. (2001). A sperm ion channel required for sperm motility and male fertility. Nature 413, 603-609. doi: 10.1038/35098027

Rosado-Berrios, C. A., Vélez, C., and Zayas, B. (2011). Mitochondrial permeability and toxicity of diethylhexyl and monoethylhexyl phthalates on TK6 human lymphoblasts cells. Toxicol. In Vitro 25, 2010-2016. doi: 10.1016/j.tiv.2011.0 8.001

Roth, Z. (2018). Symposium review: reduction in oocyte developmental competence by stress is associated with alterations in mitochondrial function 1 . J. Dairy Sci. 101, 3642-3654. doi: 10.3168/jds.2017-13389

Rudel, R. A., Camann, D. E., Spengler, J. D., Korn, L. R., and Brody, J. G. (2003). Phthalates, alkylphenols, pesticides, polybrominated diphenyl ethers, and other endocrine-disrupting compounds in indoor air and dust. Environ. Sci. Technol. 37, 4543-4553. doi: 10.1021/es0264596 
Saleh, R. A., and Agarwal, A. (2002). Oxidative stress and male infertility: from research bench to clinical practice. J. Androl. 23, 737-752. doi: 10.1002/j.19394640.2002.tb02324.x

Salicioni, A. M., Platt, M. D., Wertheimer, E. V., Arcelay, E., Allaire, A., Sosnik, J., et al. (2007). Signalling pathways involved in sperm capacitation. Soc. Reprod. Fertil. Suppl. 65, 245-259.

Schaedlich, K., Gebauer, S., Hunger, L., Beier, L.-S., Koch, H. M., Wabitsch, M., et al. (2018). DEHP deregulates adipokine levels and impairs fatty acid storage in human SGBS-adipocytes. Sci. Rep. 8:3447. doi: 10.1038/s41598-018-21800-4

Schiffer, C., Müller, A., Egeberg, D. L., Alvarez, L., Brenker, C., Rehfeld, A., et al. (2014). Direct action of endocrine disrupting chemicals on human sperm. EMBO Rep. 15, 758-765. doi: 10.15252/embr.201438869

Sepideh, J., Reza, S. M., Mahdi, A. M., Azadeh, E. H., Naser, A., Niknam, L., et al. (2009). Tyrosine phosphorylation pattern in sperm proteins isolated from normospermic and teratospermic men. J. Reprod. Infertil. 10, 185-191.

Sikka, S. (2001). Relative impact of oxidative stress on male reproductive function. Curr. Med. Chem. 8, 851-862. doi: 10.2174/0929867013373039

Sumner, R. N., Tomlinson, M., Craigon, J., England, G. C. W., and Lea, R. G. (2019). Independent and combined effects of diethylhexyl phthalate and polychlorinated biphenyl 153 on sperm quality in the human and dog. Sci. Rep. 9:3409. doi: 10.1038/s41598-019-39913-9

Sun, Y., Irie, M., Kishikawa, N., Wada, M., Kuroda, N., and Nakashima, K. (2004). Determination of bisphenol A in human breast milk by HPLC with columnswitching andfluorescence detection. Biomed. Chromatogr. 18, 501-507. doi: 10.1002/bmc. 345

Svechnikova, I., Svechnikov, K., and Söder, O. (2007). The influence of di-(2ethylhexyl) phthalate on steroidogenesis by the ovarian granulosa cells of immature female rats. J. Endocrinol. 194, 603-609. doi: 10.1677/JOE-07-0238

Tamburrino, L., Marchiani, S., Minetti, F., Forti, G., Muratori, M., and Baldi, E. (2014). The CatSper calcium channel in human sperm: relation with motility and involvement in progesterone-induced acrosome reaction. Hum. Reprod. 29, 418-428. doi: 10.1093/humrep/det454

Tanaka, A., Adachi, T., Takahashi, T., and Yamaha, T. (1975). Biochemical studies on phthalic esters I. Elimination, distribution and metabolism of di-(2-ethylhexyl)phthalate in rats. Toxicology 4, 253-264. doi: 10.1016/0300483X(75)90105-5

Toft, G., Jönsson, B. A. G., Lindh, C. H., Jensen, T. K., Hjollund, N. H., Vested, A., et al. (2012). Association between pregnancy loss and urinary phthalate levels around the time of conception. Environ. Health Perspect. 120, 458-463. doi: 10.1289/ehp.1103552

Tripathi, A., Pandey, V., Sahu, A. N., Singh, A., and Dubey, P. K. (2019). Di-(2-ethylhexyl) phthalate (DEHP) inhibits steroidogenesis and induces mitochondria-ROS mediated apoptosis in rat ovarian granulosa cells. Toxicol. Res. 8, 381-394. doi: 10.1039/c8tx00263k

Vandenberg, L. N., Hauser, R., Marcus, M., Olea, N., and Welshons, W. V. (2007). Human exposure to bisphenol A (BPA). Reprod. Toxicol. 24, 139-177. doi: 10.1016/j.reprotox.2007.07.010

Vergara, G. J., Irwin, M. H., Moffatt, R. J., and Pinkert, C. A. (1997). In vitro fertilization in mice: strain differences in response to superovulation protocols and effect of cumulus cell removal. Theriogenology 47, 1245-1252. doi: 10.1016/ S0093-691X(97)00104-0

Villegas, J., Kehr, K., Soto, L., Henkel, R., Miska, W., and Sánchez, R. (2003). Reactive oxygen species induce reversible capacitation in human spermatozoa. Andrologia 35, 227-232. doi: 10.1046/j.1439-0272.2003.00564.x

Visconti, P. E. (2009). Understanding the molecular basis of sperm capacitation through kinase design. Proc. Natl. Acad. Sci. U.S.A. 106, 667-668. doi: 10.1073/ pnas.0811895106

Visconti, P. E., Bailey, J., Moore, G., Pan, D., Olds-Clarke, P., and Kopf, G. (1995a). Capacitation of mouse spermatozoa I. Correlation between the capacitation state and protein tyrosine phosphorylation. Development 121, 1129-1137.
Visconti, P. E., Moore, G., Bailey, J., Leclerc, P., Connors, S., Pan, D., et al. (1995b). Capacitation of mouse spermatozoa. II. Protein tyrosine phosphorylation and capacitation are regulated by a cAMP-dependent pathway. Development 121, 1139-1150.

vom Saal, F. S., Parmigiani, S., Palanza, P. L., Everett, L. G., and Ragaini, R. (2008). The plastic world: sources, amounts, ecological impacts and effects on development, reproduction, brain and behavior in aquatic and terrestrial animals and humans. Environ. Res. 108, 127-130. doi: 10.1016/j.envres.2008. 03.008

Wang, W., Craig, Z., Basavarajappa, M. S., Hafner, K. S., and Flaws, J. A. (2012). Mono-(2-Ethylhexyl) phthalate induces oxidative stress and inhibits growth of mouse ovarian antral follicles1. Biol. Reprod. 87:152. doi: 10.1095/biolreprod. 112.102467

Wang, Y., Zhu, H., and Kannan, K. (2019). A review of biomonitoring of phthalate exposures. Toxics 7:21. doi: 10.3390/TOXICS7020021

Wathes, D. C., Abayasekara, D. R., and Aitken, R. J. (2007). Polyunsaturated fatty acids in male and female reproduction. Biol. Reprod. 77, 190-201. doi: 10.1095/ biolreprod.107.060558

Wennemuth, G., Carlson, A. E., Harper, A. J., and Babcock, D. F. (2003). Bicarbonate actions on flagellar and $\mathrm{Ca} 2+$-channel responses: initial events in sperm activation. Development 130, 1317-1326. doi: 10.1242/dev.00353

Williams, A. C., and Ford, W. C. L. (2005). Relationship between reactive oxygen species production and lipid peroxidation in human sperm suspensions and their association with sperm function. Fertil. Steril. 83, 929-936. doi: 10.1016/j. fertnstert.2004.11.031

Wu, Y., Li, K., Zuo, H., Yuan, Y., Sun, Y., and Yang, X. (2014). Primary neuronalastrocytic co-culture platform for neurotoxicity assessment of di-(2-ethylhexyl) phthalate. J. Environ. Sci. 26, 1145-1153. doi: 10.1016/S1001-0742(13)60 504-5

Yanagimachi, R. (1994). Fertility of mammalian spermatozoa: its development and relativity. Zygote 2, 371-372. doi: 10.1017/S0967199400002240

Yanagimachi, R. (2011). Mammalian sperm acrosome reaction: where does it begin before fertilization? Biol. Reprod. 85, 4-5. doi: 10.1095/biolreprod.111.092601

Zalata, A. A., Ahmed, A. H., Allamaneni, S. S. R., Comhaire, F. H., and Agarwal, A. (2004). Relationship between acrosin activity of human spermatozoa and oxidative stress. Asian J. Androl. 6, 313-318.

Zhou, W., Fang, F., Zhu, W., Chen, Z. J., Du, Y., and Zhang, J. (2017). Bisphenol $\mathrm{A}$ and Ovarian reserve among infertile women with polycystic Ovarian syndrome. Int. J. Environ. Res. Public Health 14:18. doi: 10.3390/ijerph1401 0018

Zota, A. R., Calafat, A. M., and Woodruff, T. J. (2014). Temporal trends in phthalate exposures: findings from the National Health and Nutrition Examination Survey, 2001-2010. Environ. Health Perspect. 122, 235-241. doi: 10.1289/ehp. 1306681

Zota, A. R., Phillips, C. A., and Mitro, S. D. (2016). Recent fast food consumption and bisphenol A and phthalates exposures among the U.S. population in NHANES, 2003-2010. Environ. Health Perspect. 124, 1521-1528. doi: 10.1289/ ehp. 1510803

Conflict of Interest: The authors declare that the research was conducted in the absence of any commercial or financial relationships that could be construed as a potential conflict of interest.

Copyright (c) 2020 Khasin, Della Rosa, Petersen, Moeller, Kriegsfeld and Lishko. This is an open-access article distributed under the terms of the Creative Commons Attribution License (CC BY). The use, distribution or reproduction in other forums is permitted, provided the original author(s) and the copyright owner(s) are credited and that the original publication in this journal is cited, in accordance with accepted academic practice. No use, distribution or reproduction is permitted which does not comply with these terms. 